\title{
Search for new phenomena in high-mass diphoton final states with ATLAS at $\sqrt{s}=13 \mathrm{TeV}$
}

\author{
Thomas Meideck, on behalf the ATLAS Collaboration \\ LPSC, Université Grenoble-Alpes, CNRS/IN2P3, Grenoble, France \\ E-mail: thomas.meideck@lpsc.in2p3.fr
}

\begin{abstract}
After the discovery of the Higgs boson, one of the most important tasks of high energy physics at the LHC is to look for signatures of new physics beyond the Standard Model. Various extensions of the Standard Model predict the existence of new high-mass states that decay into two photons, and the experimental observation of such states would be a first evidence of new physics. This search is currently ongoing in the ATLAS experiment at the Large Hadron Collider. The analysis is based on proton-proton collision data corresponding to an integrated luminosity of $36.7 \mathrm{fb}^{-1}$ at $\sqrt{\mathrm{s}}=13 \mathrm{TeV}$ recorded in 2015 and 2016. Two different studies are performed, one targeted at the search for a spin-2 particle, using Randall-Sundrum or ADD extra-dimension model as benchmark models, and one optimized for a spin- 0 particle. The observed diphoton invariant mass spectrum in both studies is in good agreement with the expected Standard Model background.
\end{abstract}

EPS-HEP 2017, European Physical Society conference on High Energy Physics

5-12 July 2017

Venice, Italy 


\section{Introduction}

Several models try to resolve the Standard Model (SM) limitations, such as the hierarchy problem. Some of them predict new high-mass states that decay into two photons. In the analysis presented here and detailed in Ref. [1], evidence of new states is searched for, in the context of an extended Higgs sector [2]-[3] and of two extra-dimensional models: the RS1 model (RandallSundrum [4]) and the ADD model (Arkani-Hamed, Dimopoulos and Dvali [5]. This analysis is performed with an integrated luminosity of $36.7 \mathrm{fb}^{-1}$ at $\sqrt{\mathrm{s}}=13 \mathrm{TeV}$ recorded in 2015 and 2016 in the ATLAS experiment at the Large Hadron Collider.

Experimentally, the different models of an extended Higgs sector predicting a heavier scalar boson can be observed by looking for a resonance in the diphoton invariant mass spectrum. This resonance can be described with two parameters, the mass of the new particle $\mathrm{m}_{\mathrm{X}}$ and the resonnance width $\Gamma_{\mathrm{X}}$.

In the RS1 model, one warped extra dimension is postulated. This gives a 5-dimensional space-time where only the gravity can propagate in the added dimension, while the SM fields are constrained to the usual space. The apparent Planck mass in this space-time dimension is given by the relation $\Lambda_{\Pi}=\bar{M}_{P l} \exp (-k \pi R)$ where $\bar{M}_{P l}$ is the Planck mass in the usual 4D space-time divided by $8 \pi, k$ is the curvature of the fifth dimension and $R$ is the compactification radius. The problem of the hierarchy can be solved if $k R \simeq 11$, which leads to a series of Kaluza Klein (KK) excitations, predicted to be at the $\mathrm{TeV}$ scale. In this paper the RS1 model parameters are expressed using the mass $\mathrm{m}_{\mathrm{G}}$ of the lightest KK excitation of the graviton and the coupling to SM fields $k / \bar{M}_{P l}$. Experimentally, KK excitations decaying into two photons can be observed as resonances on the diphoton invariant mass spectrum. In the ADD model, $n$ flat additional spacial dimensions (whose access is also restricted to gravity) are added (all dimensions with the same compactification radius $R$ ). This leads to a continuous mass spectrum of the graviton excitations. If the ultraviolet cutoff scale $\left(\mathrm{M}_{\mathrm{S}}\right)$ of the KK spectrum is lower than the Planck scale in the $(4+n)$-dimensional space-time, the extra dimensions may be detected via virtual KK graviton exchange instead of being observed via direct KK graviton emission. Experimentally, an ADD signal is observed as a continuum in the invariant mass spectrum below the mass $\mathrm{M}_{\mathrm{S}}$. The expression of $\mathrm{M}_{\mathrm{S}}$ depends on the formalism (GRW [6], Hewett [7] or HLZ [8]). The ADD model parameters can be expressed using $n$ and $\mathrm{M}_{\mathrm{S}}$.

\section{Data selection and background composition}

The analysis presented here looks for final states with two photons. Their energy deposit is measured by the Liquid Argon electromagnetic calorimeter of the ATLAS detector. A complete description of the ATLAS detector can be found in [9].

For both studies (spin-0 states and spin-2 states studies), the events are recorded using a trigger requiring at least one photon candidate with a transverse energy $E_{\mathrm{T}}^{\gamma}>35 \mathrm{GeV}$ and a second photon candidate with a transverse energy $E_{\mathrm{T}}^{\gamma}>25 \mathrm{GeV}$. Futhermore, they should satisfy identification requirements based on the leakage of energy into the hadronic calorimeter and the transverse width of the electromagnetic shower. This trigger is fully efficient for high mass diphoton events passing the following offline selection requirements. Events are then required to have at least one primary collision vertex, with at least three reconstructed tracks. At least two photons are required 
to be in the precision region of the electromagnetic calorimeter defined by $0<|\eta|<1.37$ and $1.52<|\eta|<2.37$, and to satisfy standard quality criteria. In addition these photons need to pass additional identification requirements, based again on the leakage of energy into the hadronic calorimeter and the transverse width of the electromagnetic shower, but tighter than the requirements used in the trigger. These requirements allow to further discriminate photons and jets and to obtain a better purity in the selected photon sample. The searches for spin- 0 and spin- 2 particles exploit different kinematic properties of the photons. In the selection used to search for a spin- 0 resonance (spin- 0 selection) the transverse energy $E_{\mathrm{T}}$ of the most energetic photon is required to be $E_{\mathrm{T}}>0.4 m_{\gamma \gamma}$ and the transverse energy of the second one is required to be $E_{\mathrm{T}}>0.3 m_{\gamma \gamma}$, where $m_{\gamma \gamma}$ is the diphoton invariant-mass. In the spin-2 selection, the two most energetic photons are required to have a tranverse energy larger than $55 \mathrm{GeV}$. The next requirement uses the isolation transverse energy variable $\left(E_{\mathrm{T}}^{\text {iso }}\right)$ which is calculated for each photon by summing the tranverse energy over the cells within a cone of radius $\Delta R=\sqrt{\left(\eta-\eta_{\gamma}\right)^{2}+\left(\phi-\phi_{\gamma}\right)^{2}}<0.4$ around the direction of the photon and subtracting the transverse energy of the photon itself. In addition, a soft jet contribution from underlying events is subtracted to the energy deposit. Each photon is required to have $E_{\mathrm{T}}^{\text {iso }}<2.5 \mathrm{GeV}$ to further increase the purity of the selected photon sample. Finally, the last requirement concerns the photon track isolation variable, which is computed by taking the scalar sum of the transverse momenta $p_{\mathrm{T}}$ of the tracks in a cone of size $\Delta R=0.2$ around the photon candidate.

Four different backgrounds contribute to the total diphoton SM background: one irreducible background composed of $\gamma-\gamma$ events and three reducible backgrounds with $\gamma$-jet, jet- $\gamma$ and jet-jet events where one or two jets are misidentified as photons. Three different methods, detailed in Ref. [10], have been used to study the background composition with compatible results: $2 \times 2 \mathrm{D}$ sideband method, matrix method and 2D isolation template fit method.

\section{Background estimation}

The background estimation in the spin- 0 search is done by fitting the $\gamma-\gamma$ invariant mass distribution, show in Figure 1 (left), using a smooth functional form, with parameter values determined in situ to model the total background. This functional form is tested beforehand using a diphoton SHERPA $\gamma-\gamma$ MC sample mixed with a data-driven reducible background component obtained using control samples. The mixture is based on the background composition studies. The background estimation is performed for $180<m_{\gamma \gamma}<2700 \mathrm{GeV}$. The systematic uncertainty on the background estimation is given by the fitted signal yield ('spurious signal'). The spurious signal is required to be less than $30 \%$ of the statistical uncertainty in the fitted signal yield (from the background distribution) and less than $50 \%$ if $m_{\gamma \gamma}<500 \mathrm{GeV}$ for the functional form to be validated.

In the spin-2 resonance search and also in the non-resonant signal search, the shape of the invariant mass spectrum of the irreducible background is simulated using PYTHIA, then reweighted taking into account DIPHOX NLO cross-sections. The shapes of the reducible backgrounds are fitted in control regions where the identification requirement has been inverted for one or both photons. Then the different background components are combined according to the background composition studies. The background estimation is performed for $m_{\gamma \gamma}>200 \mathrm{GeV}$ in both resonant and non-resonant signal searches, and is shown Figure 1 (right) with the $\gamma-\gamma$ invariant mass distribution. The systematic uncertainties on the background estimation arise from three different 
sources: the irreducible background uncertainties obtained by varying the renormalization and the factorization scales and the PDFs, the background composition and the uncertainties on the fits of the reducible backgrounds.
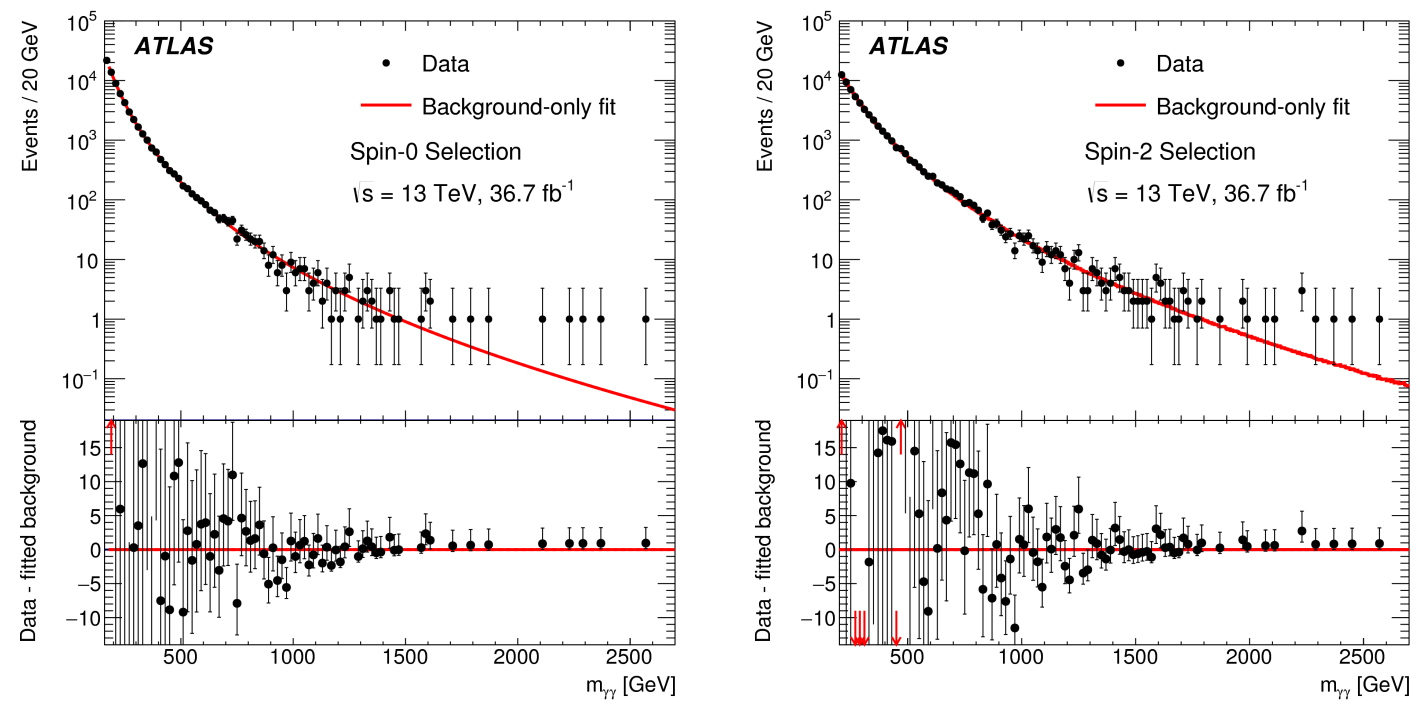

Figure 1: Distributions of the diphoton invariant mass for events passing (left) the spin-0 selection and (right) the spin-2 selection, with the background-only fits superimposed [1].

\section{Results and interpretation}

The largest local significance in the spin- 0 analysis is $2.6 \sigma$ at a mass hypothesis $\mathrm{m}_{\mathrm{X}}=730 \mathrm{GeV}$ and with a narrow width approximation. In the spin-2 resonant analysis, the largest local significance is $3.0 \sigma$ at a mass hypothesis $\mathrm{m}_{\mathrm{G}}=708 \mathrm{GeV}$ and with a coupling $k / \bar{M}_{P l}=0.1$. Nonetheless the global signal significances are respectively null and $0.8 \sigma$. In the absence of any significant deviation, 95\% CL upper limits are set on the spin-0 signal fiducial cross sections and on the resonant spin-2 signal production cross sections (Figure 2). For the non-resonant search, a counting experiment in the region $m_{\gamma \gamma}>2240 \mathrm{GeV}$ has been performed, setting a 95\% CL upper limit on the number of ADD signal events (Figure 3). These upper limits for both spin-2 signals are converted into an exclusion limit for the parameters $\mathrm{m}_{\mathrm{G}}$ and $\mathrm{M}_{\mathrm{S}}$. The exclusion limit on the parameters $\mathrm{m}_{\mathrm{G}}$ in the RS1 model with a coupling hypothesis $k / \bar{M}_{P l}=0.1$ is set to $4.1 \mathrm{TeV}$. Table 1 summarizes the different exclusion limits for the parameter $\mathrm{M}_{\mathrm{S}}$ in different ADD formalisms.

\begin{tabular}{|c|l||c|c|cccc|}
\hline & ADD formalism & GRW & Hewett & \multicolumn{4}{|c|}{ HLZ } \\
& Parameter & & positive & $n=3$ & $n=4$ & $n=5$ & $n=6$ \\
\hline \hline Without K-factor & $\mathrm{M}_{\mathrm{S}}$ observed limit $[\mathrm{TeV}]$ & 6.8 & 6.1 & 8.1 & 6.8 & 6.1 & 5.7 \\
\hline With K-factor & $\mathrm{M}_{\mathrm{S}}$ observed limit $[\mathrm{TeV}]$ & 7.2 & 6.5 & 8.6 & 7.2 & 6.5 & 6.1 \\
\hline
\end{tabular}

Table 1: Observed 95\% CL lower limits on the ADD model parameter $M_{\mathrm{S}}$ for different formalisms [1]. A K-factor was computed using ADD samples generated at LO and NLO using MADGRAPH5_AMC@ NLO. 

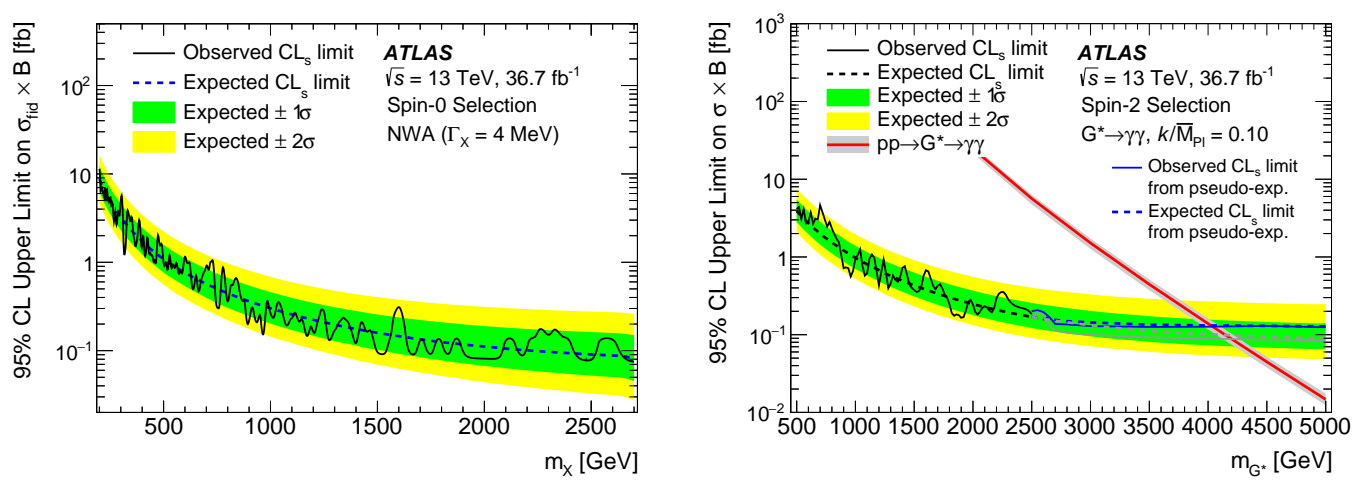

Figure 2: (left) Upper limits on the fiducial cross-section of a narrow-width spin-0 particle. (right) Upper limits of the production cross-section on the lightest RS graviton with a coupling hypothesis $k / \bar{M}_{P l}=0.1$ [1].

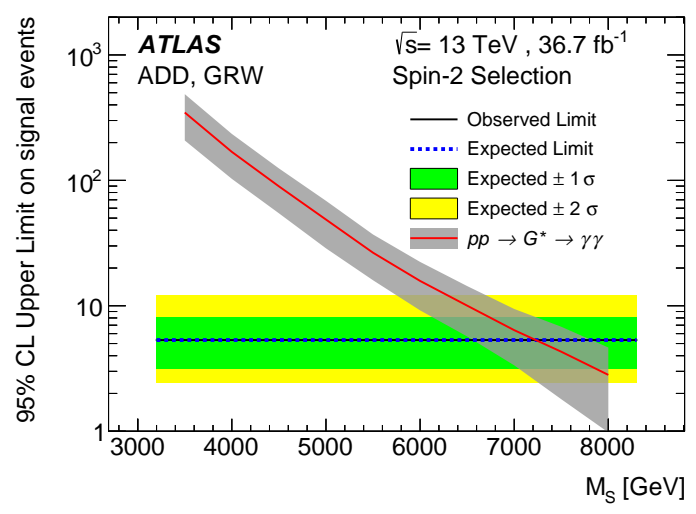

Figure 3: Upper limits on the number of ADD signal events [1].

\section{References}

[1] ATLAS Collaboration, arXiv:1707.04147 (2017).

[2] A. Hill and J.J. van der Bij, Phys. Rev. D36 3463 (1987).

[3] R. Schabinger and J. D. Wells, Phys. Rev. D72 093007 (2005).

[4] L. Randall and R. Sundrum, Phys. Rev. Lett. 83, 3370 (1999).

[5] N. Arkani-Hamed, S. Dimopoulos, and G.R. Dvali, Phys. Lett. B429, 263 (1998).

[6] G. Giudice, et al., Nucl. Phys. B544, 3 (1999).

[7] J. Hewett, Phys. Rev. Lett. 82, 4765 (1999).

[8] T. Han, et al., Phys. Rev. D59, 105006 (1999).

[9] ATLAS Collaboration, JINST 3, S08003 (2008).

[10] ATLAS Collaboration, JHEP 09001 (2016). 